\title{
Role of strain and ligand effects in the modification of the electronic and chemical properties of bimetallic surfaces
}

Kitchin, J.R.; Nørskov, Jens Kehlet; Barteau, M.A.; Chen, J.G.

Published in:

Physical Review Letters

Link to article, DOI:

10.1103/PhysRevLett.93.156801

Publication date:

2004

Document Version

Publisher's PDF, also known as Version of record

Link back to DTU Orbit

Citation $(A P A)$ :

Kitchin, J. R., Nørskov, J. K., Barteau, M. A., \& Chen, J. G. (2004). Role of strain and ligand effects in the modification of the electronic and chemical properties of bimetallic surfaces. Physical Review Letters, 93(15), 156801. https://doi.org/10.1103/PhysRevLett.93.156801

\section{General rights}

Copyright and moral rights for the publications made accessible in the public portal are retained by the authors and/or other copyright owners and it is a condition of accessing publications that users recognise and abide by the legal requirements associated with these rights.

- Users may download and print one copy of any publication from the public portal for the purpose of private study or research.

- You may not further distribute the material or use it for any profit-making activity or commercial gain

- You may freely distribute the URL identifying the publication in the public portal 


\title{
Role of Strain and Ligand Effects in the Modification of the Electronic and Chemical Properties of Bimetallic Surfaces
}

\author{
J. R. Kitchin, ${ }^{1}$ J. K. Nørskov, ${ }^{2}$ M. A. Barteau, ${ }^{1}$ and J. G. Chen ${ }^{1}$ \\ ${ }^{1}$ Center for Catalytic Science and Technology, Department of Chemical Engineering, University of Delaware, \\ Newark, Delaware 19716, USA \\ ${ }^{2}$ Center for Atomic-scale Materials Physics and Department of Physics, Technical University of Denmark, \\ DK-2800 Lyngby, Denmark
}

(Received 12 May 2004; published 4 October 2004)

\begin{abstract}
Periodic density functional calculations are used to illustrate how the combination of strain and ligand effects modify the electronic and surface chemical properties of $\mathrm{Ni}, \mathrm{Pd}$, and $\mathrm{Pt}$ monolayers supported on other transition metals. Strain and the ligand effects are shown to change the width of the surface $d$ band, which subsequently moves up or down in energy to maintain a constant band filling. Chemical properties such as the dissociative adsorption energy of hydrogen are controlled by changes induced in the average energy of the $d$ band by modification of the $d$-band width.
\end{abstract}

PACS numbers: 73.20.At, 71.20.Be

Numerous surface science studies have shown that the chemisorption properties of supported monolayers of one metal on another metal can be dramatically different than those of the parent metals [1-3]. There are two primary mechanisms for modification of the chemical properties of these surfaces. First, the average bond lengths between the metal atoms in the supported monolayer surface are typically different than those in the parent metals, resulting in changes due to strain. Second, heterometallic bonding interactions, termed the "ligand effect", between the surface atoms and the substrate can result in modification of the surface electronic structure, thereby changing the surface chemical properties. However, it has been difficult to separate the strain and ligand effects, even in surface science studies, because they usually occur together. In other words, one typically observes the cumulative effect of the two phenomena. One notable recent success was the deconvolution of these two effects for heteroepitaxial layers of $\mathrm{Pt}$ on $\mathrm{Ru}(0001)$ using a combination of experiments and theory [4], but this work succeeded only in explaining the behavior of a single system.

Mavrikakis et al. used density functional (DFT) calculations to show that strain modifies the chemical properties of surfaces by changing the average energy of the $d$ band [5]. DFT was also recently used to illustrate the ligand effect, by showing that the presence of subsurface $3 \mathrm{~d}$ metals in $\mathrm{Pt}(111)$ surfaces broadens the $\mathrm{Pt}$ surface $d$ band. The width of the surface $d$ band was found to be proportional to the interatomic matrix element that describes bonding interactions between the $d$ orbitals of the Pt surface atoms and the $d$ orbitals of the subsurface $3 \mathrm{~d}$ metal atoms [6]. As a consequence of the band broadening, the average energy of the $d$ band moved down in energy in order to conserve the $d$-band filling, resulting in modifications of the surface chemical properties.
In the current Letter, we present a simple explanation for the electronic modifications induced by strain and ligand effects in bimetallic surfaces, as well as the effects of these modifications on the chemical properties of these surfaces. We will show that the width of the $d$ band is modified by both strain and the ligand effect, and that the effects of these two mechanisms are cumulative. The $d$-band width is found to be proportional to the interatomic matrix element describing bonding between the $d$ orbitals of an atom and the $d$ orbitals of its nearest neighbors. The average energy of the $d$ band is increased or decreased depending on whether the band becomes narrower or wider due to the combination of strain and ligand effects to maintain a constant $d$-band filling. Finally, changes in chemical properties of these bimetallic surfaces due to the combined strain and ligand effects can be traced to changes in the average energy of the surface $d$ band associated with the changes in $d$-band width.

The supported monolayer calculations were performed using DACAPO on three layer slabs of the closest-packed surface for the substrate crystal structure [fcc(111), bcc(110), or hcp(0001)]. The surface layer of the slab was replaced with the monolayer metal in the bulk truncated position. In our notation, PtW designates a pseudomorphic monolayer of $\mathrm{Pt}$ on a $\mathrm{W}(110)$ surface, $\mathrm{NiRu}$ designates a pseudomorphic Ni monolayer on $\mathrm{Ru}(0001)$, and NiPt designates a pseudomorphic Ni monolayer on $\mathrm{Pt}(111)$. Relaxation of the slab was allowed in some cases, and the effects of this are discussed later. The slabs were periodically repeated in $2 \times 2$ super cells, and the ionic cores were represented by Vanderbilt ultrasoft pseudopotentials. The Kohn-Sham one-electron orbitals were expanded using planewaves up to a $350 \mathrm{eV}$ cutoff energy. The Brillouin zone was sampled either by a Chadi-Cohen grid of $18 \mathrm{k}$ points or a $4 \times 4 \times 1$ Monkhorst-Pack grid. The trends and conclusions in this work were independent 
of this choice. The PW91 functional was used to describe exchange and correlation. Properties of the $d$ band were calculated by projecting the Kohn-Sham orbitals onto spherical harmonics centered on the atomic sites.

We first examine whether the formation of the bimetallic surfaces alters the filling of the surface $d$ band. Changes in the $d$-band filling would indicate charge transfer between the two metals, and could be one of the mechanisms for chemical modification. In all cases, the change in $d$-band filling was typically less than $2 \%$ in magnitude on formation of the bimetallic surface. We interpreted these as negligible changes. Furthermore, the magnitude and sign of the change is basis set dependent; the magnitudes decrease and the sign can change if a cutoff radius is used to limit overcounting from neighboring sites. Thus, these changes may not be meaningful, aside from the observation that they are small. We also found that the $d$-band filling does not change when strain is artificially introduced despite strain-induced changes in the $d$-band width.

As shown for a rectangular band model, if the $d$-band filling remains constant but the $d$-band width changes, then the average energy of the $d$ band (the $d$-band center) must change to conserve both the $d$-band filling and the total number of $d$ states [6]. Figure 1 shows the correlation between the root mean squared (rms) $d$-band width and the $d$-band center for both unrelaxed and relaxed slabs. The slope of the correlation is nearly -1 , as predicted by the rectangular band model. Thus, as the $d$ band becomes wider it moves down in energy, and as it narrows it moves up in energy. The nonzero intercept is likely an artifact of the gaussian broadening used in the projection of the density of states onto atomic orbitals to account for the finite number of $\mathrm{k}$ points used in the calculations. This

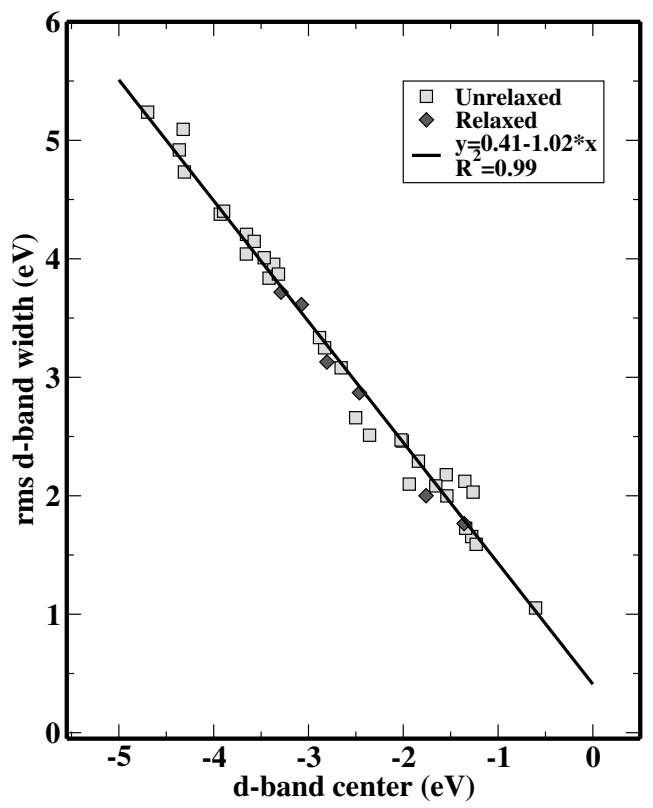

FIG. 1. Correlation between the root mean squared (rms) $d$-band width and $d$-band center for bimetallic surfaces. prevents the $d$-band width from going to zero in the atomic limit as it should.

The width of the $d$ band can be related to the interatomic matrix element that describes bonding between an atom and its environment [7]. The matrix element describing the bonding interactions between the $d$ states on two atoms of type one and type two is given by [7]

$$
V_{d d m}^{(1,2)}=\eta_{d d m} \frac{\hbar^{2}\left[r_{d}^{(1)} r_{d}^{(2)}\right]^{3 / 2}}{m d_{1,2}^{5}}
$$

where $m$ is the mass of an electron, and $r_{d}^{(i)}$ is a characteristic length for metal $i$ that is related to the spatial extent of the $d$ orbitals of that metal, which was obtained from the Solid State Table in Ref. [7]. The general trends in this Table show that $r_{d}^{(i)}$ increases from right to left and from top to bottom among the transition metals in the Periodic Table. Alternatively, $r_{d}^{(i)}$ can be shown to be proportional to the linear muffin tin orbital (LMTO) potential parameters by $r_{d}^{(i)} \propto M_{i d}^{2 / 3} \propto\left(s_{i}^{5} \Delta_{d}^{\text {Andersen }}\right)^{1 / 3}[8,9]$. Here $M_{i d}$ is an intra-atomic matrix element, $s_{i}$ is the experimental Wigner-Seitz radius and $\Delta_{d}^{\text {Anderson }}$ is a potential parameter, both of which can be found in Ref. [10]. Ruban et al. reported coupling matrix elements $V_{a d}^{2}$, which are proportional to $M_{i d}^{2}$, between an adsorbate state and a metal $d$ state for all of the transition metals [9]. Thus, Eq. (1) is seen to be equivalent to Eq. 6 in Ref. [9], but applied specifically to the coupling between two $d$-states. $d_{1,2}$ is the internuclear distance between atoms 1 and 2. $\eta_{d d m}$ is a dimensionless proportionality constant that is formally related to the type of $d d$ orbital bonding interaction, such as $V_{d d \sigma}, V_{d d \pi}$, or $V_{d d \delta}$. We are primarily interested in the proportionality between the interatomic matrix element and the $d$-band width, so we take $\eta_{d d m}$ to be unity for convenience, and thus treat all of the possible interactions in a lumped manner.

Equation (1) was previously used to describe the ligand effect in Pt-3d sandwich structures [6]. In that work, $d_{12}$ represented the interlayer spacing between the surface and subsurface layers. In this work, we expand upon this idea, recognizing that the ligand effect is incorporated into the numerator of Eq. (1) through the $r_{d}^{(i)}$ of each metal, and that strain is incorporated into the denominator through the distance between the atoms. We further develop the model by treating the interactions of a surface atom with all of the nearest-neighbors individually, including the subsurface nearest neighbors, so that the matrix element associated with a given surface atom is given by

$$
V_{i}=7.62 \sum_{j=1}^{N N} \frac{\left[r_{d}^{(i)} r_{d}^{(j)}\right]^{3 / 2}}{d_{i j}^{5}}
$$

where $\hbar^{2} / m=7.62 \mathrm{eV} \AA^{2}$.

According to tight binding theory, the $d$-band width should be proportional to the matrix element given by Eq. (2). This is illustrated in Fig. 2, where the rms $d$-band 


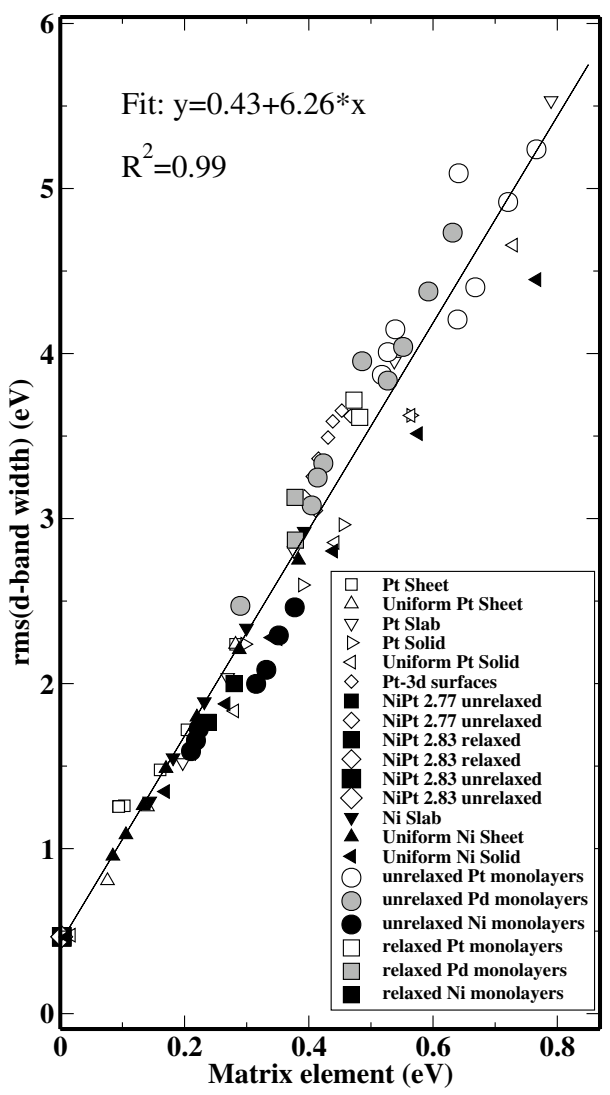

FIG. 2. Correlation between the rms $d$-band width and the matrix element. Black symbols indicate a $\mathrm{Ni}$ atom, gray symbols indicate $\mathrm{Pd}$, and white indicates $\mathrm{Pt}$. Data points labeled NiPt2.xx refer to Ni/Pt bimetallic surfaces with nearest neighbor distances of 2.77 or $2.83 \AA$ [11]. Details for the Pt-3d surfaces can be found in Ref. [6]. The keyword "Uniform" indicates the structures were strained uniformly in the unit cell lattice vector directions, otherwise, the structure was strained only in one lattice vector direction. A sheet is a single atomic layer, a slab is three layers thick and separated by five equivalent layers of vacuum. The monolayers are described in the text.

width of a large number of geometries of both pure and bimetallic $\mathrm{Ni}, \mathrm{Pd}$, and Pt-containing structures is plotted against their respective interatomic matrix elements. These structures include a variety of forms of strain, including isotropic and anisotropic strain, pseudomorphic monolayers of $\mathrm{Ni}, \mathrm{Pd}$, and $\mathrm{Pt}$ on $\mathrm{Nb}, \mathrm{Ta}, \mathrm{Mo}, \mathrm{W}, \mathrm{Re}$, $\mathrm{Ru}$, Ir, Os, Ni and Pt, surface and subsurface alloys (from Ref. [11]), bimetallic sandwich structures (from Ref. [6]), and a variety of coordination environments (sheets, slabs, and solids). Clearly, the $d$-band width is closely related to the interatomic matrix element that describes the bonding interactions between the $d$ orbitals of the atom and its nearest neighbors. We can now provide a quantititative understanding of the role of strain. In tensile strain, $d_{i j}$ is larger than in the parent metal, resulting in a smaller interatomic matrix element, and a narrower $d$ band that is higher in energy than the parent metal. In compressive strain, $d_{i j}$ is smaller than that of the parent metal, resulting in a larger interatomic matrix element, and a wider $d$ band that is lower in energy than that of the parent metal.

We conclude by showing how these electronic structure modifications are manifested in modifying the chemical properties of bimetallic surfaces. The adsorption energies and dissociative reaction barriers of many small molecules such as $\mathrm{CO}$ have been correlated with the average energy of the surface $d$ band of pure metal surfaces [12] and of alloy/overlayer surfaces [13]. We have calculated the dissociative adsorption energies for hydrogen on many of the bimetallic monolayer systems previously described in this work. In all of these calculations, the bimetallic slab was allowed to relax to the lowest energy geometry. The positions of the slab ions were subsequently frozen in their relaxed positions, and a $\mathrm{H}$ atom was placed in a three-fold site and allowed to relax to the lowest energy geometry. The dissociative adsorption energy was calculated as $\Delta H_{\text {diss,ads }}=E_{\text {slab, } \mathrm{H}}-E_{\text {slab }}-1 / 2 E_{\mathrm{H}_{2}}$. The dissociative adsorption energies are plotted against the $d$-band center of the surfaces in Fig. 3, where an obvious trend between the dissociative adsorption energy of hydrogen and the surface $d$-band center is observed. Thus, the chemical properties of these bimetallic surfaces, which include both strain and ligand effects, are modified by changes in the $d$-band center of the surface. We would like to note that a better correlation between hydrogen adsorption energies and the $d$-band center can be obtained if a cutoff radius is introduced in the projected density of states. However, the cutoff radius is arbitrary, so we have not presented these results.

Figure 3 contains bimetallic surfaces in which strain and the ligand effect work in opposite directions. For

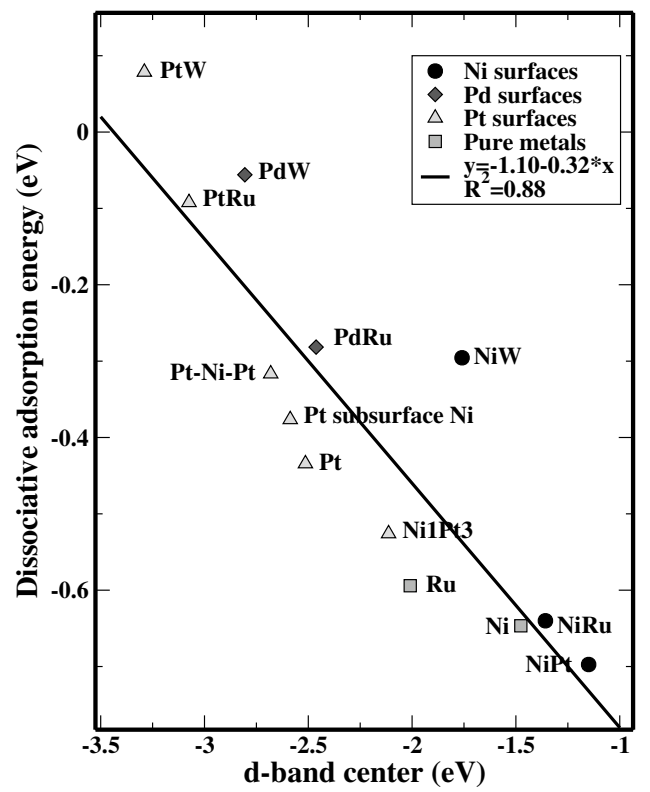

FIG. 3. Correlation between the dissociative adsorption energy of $\mathrm{H}_{2}$ on bimetallic surfaces and the $d$-band center of those surfaces. 
example, a Ni monolayer on $\mathrm{W}(110)$ is under substantial tensile strain $(>10 \%)$ compared to $\mathrm{Ni}(111)$, resulting in a narrowing of the $d$ band. However, there is a substantial broadening of the Ni $d$ band due to the strong bonding interactions with the $\mathrm{W}$ substrate. The net result is a $\mathrm{Ni}$ $d$ band that is broader and lower in energy than that of $\mathrm{Ni}(111)$ and that the dissociative adsorption energy of $\mathrm{H}_{2}$ on $\mathrm{Ni} / \mathrm{W}(110)$ is lower than that on $\mathrm{Ni}(111)$. For a $\mathrm{Ni}$ monolayer on $\mathrm{Ru}(0001)$, the tensile strain is similar to that on $\mathrm{W}(110)$, but the ligand effect is weaker. Consequently, the band sharpening effect due to strain is nearly balanced by the broadening due to the ligand effect, and the calculated electronic and chemical properties of $\mathrm{Ni} / \mathrm{Ru}(0001)$ are nearly identical to those of $\mathrm{Ni}(111)$. Finally, for a Ni monolayer on $\mathrm{Pt}(111)$, the tensile strain is again similar to that on $\mathrm{W}(110)$ or $\mathrm{Ru}(0001)$, but here the ligand effect is the weakest. Consequently, strain dominates the modification with the net effect of narrowing and raising in energy of the $\mathrm{Ni} d$ band, which results in a stronger adsorption energy on this surface. The interatomic matrix elements for each Ni surface and the corresponding $d$-band widths are tabulated in Table I. If one uses these matrix elements to predict the band widths using the fit in Fig. 2, one finds the bimetallic band widths are overestimated by $10 \%-20 \%$. The reason for this is clear from Fig. 2, in which nearly all of the Ni-containing points fall below the best fit line. This suggests that element-specific fits may give better predictive results.

Finally, the trends reported in this work hold for both unrelaxed and relaxed calculations. Allowing slabs to relax had a dramatic effect on the electronic structure and adsorption energies in some cases, but we found that both properties changed consistently, effectively moving up or down the trends accordingly. These findings are easily understood by realizing that relaxation of the slab amounts to changing the internuclear distances of the atoms in the slab, and that these changes are accounted for by the interatomic matrix elements in the denominator of Eq. (2). Thus, upon relaxation the interatomic matrix elements change, resulting in a change in the $d$-band width with a concomitant change in the $d$-band center and corresponding adsorption energy. A similar analysis could be done to estimate the effects that a surface reconstruction could have on chemical reactivity.

In conclusion, the formation of a bimetallic surface results in changes in the surface $d$-band width due to the combination of strain and ligand effects. Both of these effects are manifested in the interatomic matrix element describing bonding interactions between an atom and its nearest-neighbors. The $d$-band width and $d$-band center are highly correlated to each other due to the fact that the $d$-band filling changes negligibly upon the formation of these bimetallic surfaces. Consequently, when the combined effects result in a broader $d$ band, the average energy of the $d$ band decreases. In contrast, when the combined effects result in a more narrow $d$ band, the
TABLE I. Comparison of interatomic matrix elements and the DFT $d$-band width.

\begin{tabular}{lcc}
\hline \hline & $V$ & $W_{D F T}$ \\
\hline $\mathrm{Ni}$ & 0.23 & 1.89 \\
$\mathrm{NiPt}$ & 0.17 & 1.35 \\
$\mathrm{NiRu}$ & 0.27 & 1.76 \\
$\mathrm{NiW}$ & 0.28 & 2.00 \\
\hline \hline
\end{tabular}

average energy of the $d$ band is increased. Finally, a reasonable correlation between the dissociative adsorption energy of hydrogen and the surface $d$-band center has been demonstrated. The correlations described in the current Letter could be used as a first step towards predicting the properties of other bimetallic systems or identifying bimetallic systems with desirable electronic or chemical properties without the need to perform expensive first-principles DFT calculations. This could facilitate narrowing the focus of future studies on bimetallic or alloy surfaces.

This work was funded in part by Basic Energy Sciences, Department of Energy (Grant No. DE-FG0204ER15501). The Center for Atomic-scale Materials Physics is sponsored by the Danish National Research Foundation. The DFT calculations have been performed with support from the Danish Center for Scientific Computing through Grant No. HDW-1101-05.

[1] D.W. Goodman, Ultramicroscopy 34, 1 (1990).

[2] R. A. Campbell, J. A. Rodriguez, and D.W. Goodman, Phys. Rev. B 46, 7077 (1992).

[3] J. A. Rodriguez and D.W. Goodman, Science 257, 897 (1992).

[4] A. Schlapka, M. Lischka, A. Gro $\beta$, U. Käsberger, and P. Jakob, Phys. Rev. Lett. 91, 016101 (2003).

[5] M. Mavrikakis, B. Hammer, and J. K. Nørskov, Phys. Rev. Lett. 81, 2819 (1998).

[6] J. R. Kitchin, J. K. Nørskov, J. G. Chen, and M A. Barteau, J. Chem. Phys. 120, 10240 (2004).

[7] W. A. Harrison, Electronic Structure and the Properties of Solids (Dover Publications, Inc., New York, 1989).

[8] K.W. Jacobsen, J. K. Nørskov, and M. J. Puska, Phys. Rev. B 35, 7423 (1987).

[9] A.V. Ruban, B. Hammer, P. Stoltze, H. L. Skriver, and J. K. Nørskov, J. Mol. Catal. A: Chem. 115, 421 (1997).

[10] O. K. Andersen, O. Jepsen, and D. Glötzel, in Highlights of Condensed-Matter Theory, edited by F. Bassani, F. Fumi, and M. P. Tosi, (North-Holland, Amsterdam, 1985), Vol. LXXXIX, p. 59-193.

[11] J. R. Kitchin, N. A. Khan, J. G. Chen, M. A. Barteau, T. E. Madey, and B. Yakshinsky, Surf. Sci. 544, 295 (2003).

[12] J. K. Nørskov et al., J. Catal. 209, 275 (2002).

[13] B. Hammer, Y. Morikawa, and J. K. Nørskov, Phys. Rev. Lett. 76, 2141 (1996). 